\title{
IRRADIACIÓN LUMBOAÓRTICA EN EL CÁNCER CÉRVICO UTERINO: ANÁLISIS DE LA LITERATURA
}

\author{
Alejandro Santini B. ${ }^{1}$, Sergio Becerra S. ${ }^{1}$, Benjamín Bianchi G. ${ }^{2}$, Patricio Gayan G. ${ }^{3}$, \\ Marcela Cárcamo I. ${ }^{4}$, Daniela Seelmann G. ${ }^{a}$, Gonzalo Silva P. ${ }^{a}$, Cristóbal Besio H. ${ }^{5}$ \\ 1 Oncólogo Radioterapeuta, ${ }^{2}$ Programa de Radioterapia, ${ }^{3}$ Oncólogo Ginecólogo, ${ }^{4}$ Epidemiólogo, ${ }^{5}$ Programa de \\ Oncología Ginecológica, Instituto Nacional del Cáncer.
}

a Alumnos, Escuela de Medicina, Universidad de los Andes.

\section{RESUMEN}

Antecedentes: El cáncer de cuello uterino es una enfermedad prevalente en Chile y es una de las localizaciones tumorales más frecuentes tratadas en el Instituto Nacional del Cáncer. Es habitual recibir pacientes jóvenes con tumores avanzados, en etapas IIB y IIIB, con riesgo elevado de compromiso ganglionar, tanto pelviano como lumboaórtico y donde el tratamiento estándar es la radio-quimioterapia. Objetivos: Determinar si la irradiación lumboaórtica reduce el riesgo de recidiva y/o mejora la sobrevida en pacientes con compromiso ganglionar evidente y en aquellos con alto riesgo de compromiso a dicho nivel. Método: Revisión exhaustiva de la literatura publicada sobre la indicación de radioterapia lumboaórtica en el cáncer cérvico uterino, en pacientes con enfermedad evidente en los ganglios lumboaórticos y en aquellas en que la indicación es en casos de alto riesgo de compromiso en dicho nivel. Resultados: En pacientes con tumores cervicales de pequeño tamaño y con ganglios pelvianos positivos, sería beneficioso el tratamiento ganglionar lumboaórtico. Sin embargo, en aquellas pacientes con enfermedad pelviana masiva (IIIB) o en aquellas en que existe evidencia de enfermedad lumboaórtica, dicho beneficio no sería tan importante. Conclusión: Son las pacientes con enfermedad pelviana controlable y con elevado riesgo de tener compromiso ganglionar lumboaórtico (etapa IA-B, IIA-B con ganglios positivos en la pelvis), las que más se benefician de la radioterapia lumboaórtica.

\section{PALABRAS CLAVE: Cáncer cérvico uterino, radioterapia, revisión de la literatura}

\section{SUMMARY}

Background: Uterine cancer is a prevalent disease in Chile and it is one of the most frequent cancer locations treated in the National Chilean Cancer Institute. It is also common to receive young patients that have advanced tumors in stages IIB and IIIB with high risks of compromises of lymphatic nodes of pelvis and aortic-lumbar zones. The treatment for these kinds of cancers is radio-chemotherapy. Aims: Determinate if the radiotherapy of aortic-lumbar lymph nodes lowers the chance of relapsing or increase the survival rate in patients with evident compromise of aortic-lumbar lymph nodes and in those with high risk of compromise in that level. Method: Exhaustive analysis of the literature about the indication of radiotherapy of aortic-lumbar lymph nodes in cervix cancer which is classified in those where the radiotherapy treatment is done in patients with evident compromise of aortic-lumbar lymph-nodes, and those where de radiotherapy is done in patients with high risk of compromise in that level. Results: In patients with small cervix tumors and positive lymphoid nodes the LA lymphatic nodes treatment would be beneficial. However, patients that 
suffer massive pelvic disease (IIIB) or that have evidence of the disease, the benefit would not be so important. Conclusion: Patients with controllable pelvic disease and with high risk of having aortic-lumbar lymph nodes compromise are the most benefit of radiotherapy in aortic-lumbar lymph nodes (stages IA-B, IIA-B with positive lymph nodes).

\section{KEY WORDS: Uterine cervical cancer, radiotherapy, literature review}

\section{INTRODUCCIÓN}

El cáncer cervicouterino $(\mathrm{CaCu})$ continúa siendo una patología frecuente en los países en vías de desarrollo. Chile no es una excepción, esta enfermedad es un problema de salud nacional relevante, a pesar de que las cifras revelan una reducción en la mortalidad en los últimos años (1).

En el Instituto Nacional del Cáncer seguimos recibiendo pacientes jóvenes con tumores avanzados, donde el tratamiento fundamental es la radioterapia con o sin quimioterapia concomitante.

En los últimos años se han producido varios cambios tanto en la prevención como en el tratamiento de las pacientes con $\mathrm{CaCu}$. En el tratamiento, desde hace casi 10 años, los tumores avanzados son manejados habitualmente con la combinación de radioterapia y quimioterapia. En nuestro centro empleamos cisplatino en forma semanal (40mg/ $\mathrm{m} 3$ ) logrando un control similar a la mayoría de las series internacionales.

Los volúmenes de tratamiento con radioterapia habitualmente incluyen la totalidad del útero, los tejidos blandos periuterinos (parametrios) y los territorios ganglionares pelvianos (iliacos externos, internos y comunes). Sin embargo, el incluir los ganglios lumboaórticos se ha debatido por más de 40 años.

Existe una justificación fisiopatológica para la irradiación de los territorios ganglionares lumboaórticos que se basa en el conocimiento de la historia natural de la enfermedad. Esta neoplasia tiene una forma más o menos ordenada de diseminación ganglionar, con una secuencia habitualmente predecible, que permite estimar el riesgo de afectación de los mismos en diferentes situaciones clínicas (2). Por otro lado, está el hecho de que existen muchas pacientes en que la enfermedad se encuentra limitada a estas regiones ganglionares y en quienes el tratamiento locorregional obtiene una sobrevida prolongada e incluso curación (Tabla I) (3-10). Sin embargo, el tratamiento de los ganglios lumboaórticos implica la irradiación de una mayor cantidad de masa intestinal, así como de médula ósea, con lo cual se incrementa la toxicidad, tanto aguda como crónica a tal punto de no alcanzar una ganancia terapéutica.
Tabla I

SOBREVIDA A 5 AÑOS DE PACIENTES CON GANGLIOS LUMBOAÓRTICOS POSITIVOS TRATADAS CON RADIOTERAPIA LUMBOAÓRTICA

\begin{tabular}{lcc}
\hline Referencia, año & $\mathrm{n}$ & $\begin{array}{c}\text { Sobrevida a } \\
5 \text { años (\%) }\end{array}$ \\
\hline Hughes $^{3}, 1980$ & 22 & 29 \\
Ballon $^{4}, 1981$ & 18 & 23 \\
Piver $^{5}, 1981$ & 31 & 9,6 \\
Welander $^{6}, 1981$ & 31 & 25,8 \\
Potish $^{7}, 1985$ & 17 & 40 \\
La Polla $^{8}, 1986$ & 16 & 30 \\
Vigliotti $^{9}, 1992$ & 43 & 28 \\
Buchsbaum $^{10}, 1979$ & 21 & 23 \\
\hline TOTAL & 199 & 27,2
\end{tabular}

En los últimos trabajos publicados donde se analiza este tema se puede comprobar que existe un grupo de pacientes que se beneficiarían de dicho tratamiento $(11,12)$ Se debe considerar que con la mejoría de las técnicas actuales es factible realizar tratamientos más conformados a fin de proteger mayor cantidad de tejidos normales, logrando tratamientos mejor tolerados.

\section{DISEMINACIÓN LINFÁTICA DEL CÁNCER CER- VICOUTERINO}

La diseminación ganglionar del $\mathrm{CaCu}$ puede ser hacia cualquier territorio pelviano, incluso, aunque muy poco frecuente, directo hacia ganglios lumboaórticos. En la mayoría de los casos hay una secuencia más o menos ordenada, similar a lo que ocurre en otras localizaciones donde el carcinoma escamoso es la histología más frecuente (cáncer de laringe, cáncer de esófago)

La secuencia más habitual de diseminación es hacia los iliacos externos en primer lugar, a los iliacos comunes después y finalmente hacia los lumboaórticos. Lo vasos linfáticos nacen en dos plexos, uno a nivel de la mucosa, otro en la muscularis propia y posteriormente, a nivel de la subserosa, se juntan en los conductos colectores. Estos conductos colectores se juntan a nivel del istmo y 
constituyen tres grupos: superior, medio e inferior. El tronco colector superior surge de la zona anterior y lateral del cuello uterino, cruzan la arteria uterina y terminan en los ganglios iliacos internos altos. A dicho nivel se ha descrito con frecuencia un ganglio parauterino. Los troncos medios drenan la porción del cuello que se encuentra dentro del ligamento ancho y terminan en los ganglios obturatrices; el tronco inferior, que drena la zona más baja del cueIlo, va hacia atrás por los ligamentos útero-sacros y llega a los ganglios presacros (13). En la Tabla II se describen los lugares de origen y los territorios de drenaje de los principales troncos colectores antes mencionados (Figura 1).

En las pacientes con $\mathrm{CaCu}$ el riesgo de presentar ganglios comprometidos a nivel lumboaórtico es variable, existiendo factores de riesgo determinantes de ello. Entre ellos, el factor de mayor riesgo es la presencia de ganglios afectados en la pelvis; sin embargo, también son factores determinantes la etapa de la enfermedad, el tamaño tumoral, la presencia de invasión linfovascular y la extensión tumoral al cuerpo uterino (14).

Tabla II

\section{DRENAJE LINFÁTICO DE LOS TRONCOS COLECTORES PRINCIPALES DEL CUELLO UTERINO}

\begin{tabular}{|c|c|}
\hline Territorios de drenaje & Lugar de origen \\
\hline \multirow[t]{4}{*}{ Superior } & $\begin{array}{l}\text { Surgen de la zona anterior y la- } \\
\text { teral del cuello uterino }\end{array}$ \\
\hline & Cruzan la arteria uterina \\
\hline & $\begin{array}{l}\text { Terminan en los iliacos internos } \\
\text { altos (hipogástricos) }\end{array}$ \\
\hline & $\begin{array}{l}\text { Se ha descrito un ganglio pa- } \\
\text { rauterino (centinela) }\end{array}$ \\
\hline \multirow[t]{2}{*}{ Medio } & $\begin{array}{l}\text { Drenan la porción del cuello } \\
\text { que queda dentro del ligamen- } \\
\text { to ancho }\end{array}$ \\
\hline & $\begin{array}{l}\text { Pasa por detrás de la hipogás- } \\
\text { trica y llega a los ganglios obtu- } \\
\text { ratrices (iliacos externos bajos) }\end{array}$ \\
\hline \multirow[t]{2}{*}{ Inferior } & $\begin{array}{l}\text { Borde inferior del cuello } \\
\text { Va hacia atrás por el ligamento } \\
\text { útero- sacro y los ganglios pre- } \\
\text { sacros }\end{array}$ \\
\hline & $\begin{array}{l}\text { Algunos canales salen de la } \\
\text { cara anterior por la arteria vesi- } \\
\text { cal y llegan a los iliacos inter- } \\
\text { nos }\end{array}$ \\
\hline
\end{tabular}

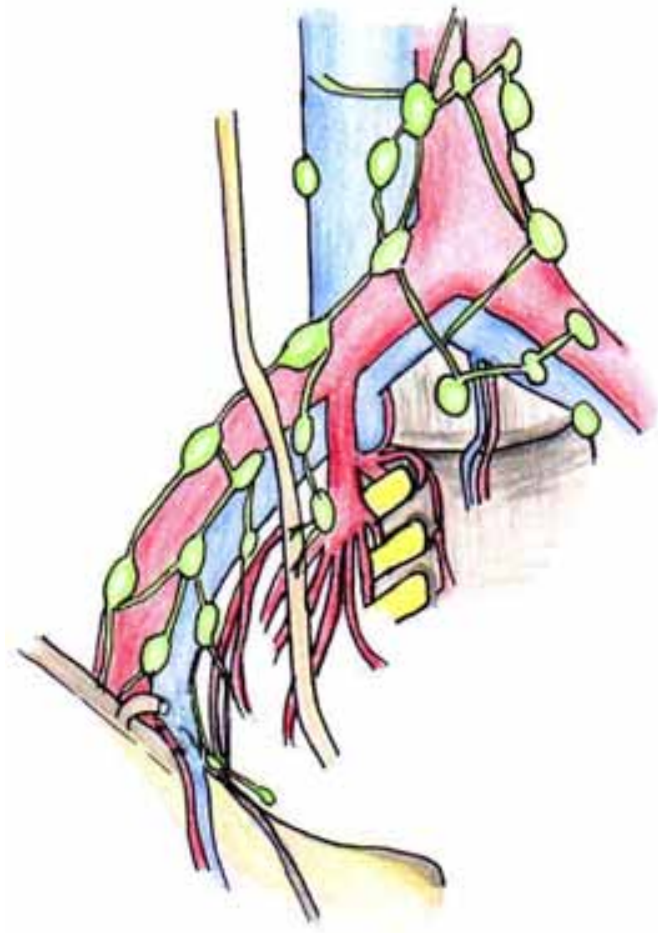

Figura 1. Drenaje linfático del cuello uterino.

La incidencia de compromiso ganglionar pelviano en las pacientes con $\mathrm{CaCu}$ es, por lo tanto, variable y relativamente predecible. Como se observa en la Tabla III, para las pacientes con una etapa IB el riesgo de ganglios pelvianos positivos es de aproximadamente $17 \%$ (15-23). En la Tabla IV se detalla el porcentaje de compromiso ganglionar lumboaórtico en pacientes con etapa II y III (16,4 y $28,6 \%$ respectivamente), según los trabajos publicados en la literatura $(3,4,7,8,10,24-29)$.

\section{TRATAMIENTO DE LAS CADENAS GANGLIO- NARES LUMBOAÓRTICAS}

Como mencionamos anteriormente, desde la década de 1960 se ha intentado controlar la enfermedad a nivel lumboaórtico, publicándose trabajos donde se realizan tratamientos con criterio profiláctico $(29,30)$ y otros en los cuales el objetivo es controlar una enfermedad evidente a dicho nivel, o sea aquellas pacientes con demostración clínica de metástasis en los ganglios lumboaórticos $(9,11,31,32)$. En estas publicaciones se demuestra que se puede obtener una sobrevida a 5 años de 51 a $67 \%$ para los tratamientos profilácticos y del $25-35 \%$ cuando el tratamiento se realiza en el contexto de una enfermedad metastásica. 
Tabla III

INCIDENCIA DE COMPROMISO GANGLIONAR PELVIANO EN CÁNCER CERVICO UTERINO ETAPA IB

\begin{tabular}{|c|c|c|}
\hline Referencias, año & $\mathrm{n}$ & $\%$ \\
\hline Fuller ${ }^{15}, 1982$ & 280 & 15 \\
\hline Finan ${ }^{16}, 1986$ & 229 & 21,4 \\
\hline Inoue ${ }^{17}, 1984$ & 362 & 13 \\
\hline Samlal ${ }^{18}, 1997$ & 271 & 19,6 \\
\hline Monagham ${ }^{19}, 1990$ & 494 & 20,6 \\
\hline Creasman ${ }^{20}, 1986$ & 258 & 14 \\
\hline Artman ${ }^{21}, 1987$ & 153 & 8,5 \\
\hline Zander $^{22}, 1981$ & 860 & 18,9 \\
\hline Timmer ${ }^{23}, 1984$ & 119 & 15,1 \\
\hline Total & 3026 & 17,3 \\
\hline
\end{tabular}

y cambia en forma significativa el manejo de las pacientes con enfermedad avanzada, permitiendo con mayor precisión la extensión de la enfermedad y de esta manera delimitar con mayor certeza los volúmenes de tratamiento (33).

En la Figura $2 \mathrm{~A}$ se observa un PET-CT de una paciente portadora de un $\mathrm{CaCu}$ donde se evidencian zonas con captación a nivel de los ganglios lumboaórticos a derecha. Esta paciente fue sometida a tratamiento de radioterapia y quimioterapia incluyendo dichos niveles ganglionares (imágenes entregadas por gentileza de Dr. F. Leborgne). En el PET-CT realizado un mes después de finalizar el tratamiento se comprueba la desaparición de dicha captación (Figura 2B). Sin embargo, el acceso a dicha técnica en nuestro servicio es más la excepción que la regla.

Uno de los primeros trabajos respecto al trata-

Tabla IV

INCIDENCIA DE COMPROMISO GANGLIONAR LUMBOAÓRTICO EN PACIENTES CON CÁNCER CERVICOUTERINO ETAPA II Y III

\begin{tabular}{|c|c|c|c|c|c|c|}
\hline \multirow[b]{2}{*}{ Referencia, año } & \multicolumn{3}{|c|}{ Etapa II } & \multicolumn{3}{|c|}{ Etapa III } \\
\hline & Examinados & + & $\%$ & Examinados & + & $\%$ \\
\hline Nelson ${ }^{24}, 1977$ & 63 & 9 & 14,3 & 39 & 15 & 38,5 \\
\hline Delgado ${ }^{25}, 1977$ & 18 & 8 & 44,4 & 13 & 5 & 38,5 \\
\hline Piver ${ }^{26}, 1977$ & 46 & 6 & 13,0 & 49 & 18 & 36,7 \\
\hline Sudarsanam ${ }^{27}, 1978$ & 43 & 7 & 16,3 & 19 & 3 & 15,8 \\
\hline Buchsbaum ${ }^{10}, 1979$ & 19 & 1 & 5,3 & 104 & 34 & 32,7 \\
\hline Hughes ${ }^{3}, 1980$ & 80 & 14 & 17,5 & 96 & 23 & 24,0 \\
\hline Ballon ${ }^{4}, 1981$ & 48 & 9 & 18,8 & 24 & 4 & 16,7 \\
\hline Welander ${ }^{6}, 1981$ & 63 & 13 & 20,6 & 38 & 10 & 26,3 \\
\hline Berman ${ }^{28}, 1984$ & 265 & 43 & 16,2 & 180 & 45 & 25 \\
\hline Potish ${ }^{7}, 1985$ & 47 & 5 & 10,6 & 11 & 4 & 36,4 \\
\hline La Polla ${ }^{8}, 1986$ & 47 & 6 & 12,8 & 38 & 14 & 36,8 \\
\hline TOTAL & 739 & 121 & 16,4 & 611 & 175 & 28,6 \\
\hline
\end{tabular}

\section{RADIOTERAPIA DE PACIENTES CON ENFER- MEDAD EVIDENTE A NIVEL LUMBOAÓRTICO}

El intento de controlar enfermedad metastásica a nivel lumboaórtica ha sido reportado en distintos trabajos que se detallan en la Tabla I. Muestran una sobrevida promedio de $27 \%$ a 5 años al utilizar radioterapia lumboaórtica en pacientes con ganglios positivos.

Un aspecto a resaltar en la actualidad es el empleo del PET (positron emission tomography) o PET-CT (positron emission tomography-computed tomography) en la evaluación de estas pacientes. Esta nueva técnica diagnóstica, ya no solo anatómica, sino fisiopatológica, abre una nueva puerta miento de aquellas pacientes con ganglios positivos fue publicado en 1974 por Vongtama y cols (34). En este trabajo se analiza el resultado del tratamiento con radioterapia en pacientes con enfermedad masiva a nivel retroperitoneal. Al momento de su publicación no se contaba con la imagenología adecuada, pero el $71 \%$ de las pacientes tenían dolor dorsal, el $55 \%$ presentaban hidroureteronefrosis y más del $30 \%$ alteraciones en la radiografía simple de columna, todos elementos clínicos de una enfermedad masiva a dicho nivel. En este grupo de pacientes aunque se planteó un tratamiento con criterio paliativo, un porcentaje no menor de pacientes lograron una sobrevida prolongada ( $8 \%$ sobrevivieron más de 72 meses). 


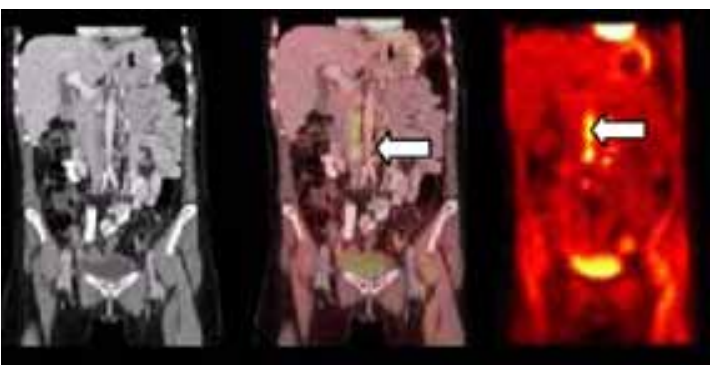

Figura 2A. PETCT de paciente con CCU con ganglios lumboaórticos positivos (flecha).

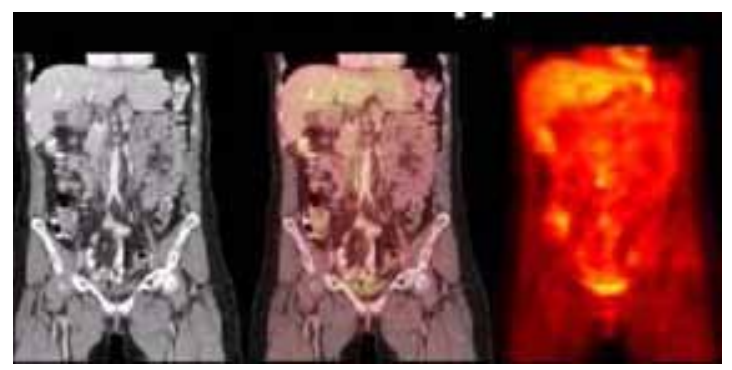

Figura $2 B$. Un mes después de finalizado el tratamiento de radioquimioterapia se confirma la desaparición de la zona hipermetabólica lumboaórtica.

Uno de los argumentos importantes en contra del tratamiento de radioterapia en pacientes con enfermedad lumboaórtica confirmada con una biopsia quirúrgica, es la elevada tasa de complicaciones. En la mayoría de los trabajos iniciales se empleaba un abordaje transperitoneal para llegar a los ganglios lumboaórticos. Luego de este tipo de cirugía la tasa de complicaciones es francamente mayor que lo que sucede luego de la cirugía hecha con técnicas mas modernas, como con el abordaje extraperitoneal $(4,35)$. Actualmente la linfadenectomía lumboaórtica se debería realizar por vía extraperitoneal y, como veremos en nuestra serie, la radioterapia postoperatoria es actualmente bastante bien tolerada.

Uno de los aspectos de mayor importancia para obtener un control de la enfermedad prolongada en pacientes con ganglios lumboaórticos comprometidos, es la extensión de la enfermedad pelviana. Varios trabajos demuestran que aquellas pacientes con una enfermedad pelviana precoz son las que obtienen un mayor beneficio del tratamiento de radioterapia lumboaórtica. Cunninham y cols (36), demuestran que aquellas pacientes en quienes en el momento de la cirugía se encuentra un compromiso ganglionar lumboaórtico, pero que tenían una enfermedad pelviana con indicación de cirugía radical, logran una sobrevida a 5 años de $48 \%$ y 6 de ellas sobrevivieron más de 10 años. Este autor comprueba que casi la mitad de las pacientes con metástasis en los ganglios lumboaórticos no tienen una enfermedad más allá de dicho nivel. En una más reciente publicación, Stryker y Mortel (32), obtienen un $30 \%$ de curación en aquellas pacientes con un cáncer cervicouterino etapa IB pero ganglios lumboaórticos positivos.

Grisgby y cols (37), analizan la probabilidad de control de la enfermedad lumboaórtica en función de la extensión de la misma, tanto a nivel pelviano como abdominal. Estos autores estudiaron la probabilidad de control ganglionar en función de su evidencia, tanto en el TAC como en el PET en 208 pacientes con CaCu etapa Ib1 a IVa. Comprobaron que la probabilidad de control tumoral con las dosis habituales de radioterapia a dicho nivel es alta y que aquellas pacientes con menos posibilidades eran las que tenían una enfermedad evidente en el TAC y PET simultáneamente. Por otro lado, la principal forma de recidiva fue a nivel sistémico. Las pacientes que más se benefician de radioterapia lumboaórtica, son aquellas con una enfermedad microscópica a nivel lumboaórtico y con una enfermedad pelviana precoz y controlable.

Otros autores han intentado también formas alternativas de radioterapia lumboaórtica, como por ejemplo el tratamiento bifraccionado; sin embargo, en estos trabajos la toxicidad fue elevada (38). Se alcanzó una dosis de hasta 58 Gy pero la toxicidad grado III y IV se dio en 17 de 29 pacientes (58\%).

\section{RADIOTERAPIA LUMBOAÓRTICA PROFILÁCTI- CA}

Los argumentos fundamentales que apoyan la irradiación profiláctica de las cadenas lumboaórticas surgen, por un lado, del hecho de que el cáncer de cuello uterino, al igual que la mayoría de los carcinomas escamosos, tiene una historia natural más o menos predecible y por etapas, con un compromiso ganglionar en primera instancia y más tarde una diseminación hematógena a distancia. Por el otro lado, una recidiva a nivel lumboaórtico es prácticamente incurable (11).

En 1979, Rotman y cols (29) publican un trabajo de radioterapia profiláctica lumboaórtica en pacientes con enfermedad IB voluminoso y IIB. Los resultados alentadores de este trabajo llevaron a la realización de un primer protocolo randomizado (RTOG 79-20) por parte del Radiation Therapy Oncology Group (RTOG), cuyos resultados se publicaron en 1990 y fueron reanalizados en 1995. En este trabajo se incluyeron 367 pacientes desde 1979 hasta 1986 con cáncer de cuello uterino etapa IB, IIA y IIB 
y sin evidencia de enfermedad lumboaórtica. Una de las ramas consistía en radioterapia pelviana sola y la otra en radioterapia pelviana y lumboaórtica. Los resultados fueron mejores en el grupo de radioterapia lumboaórtica, con una sobrevida a 10 años de $44 \%$ versus $55 \%(p<0,02)$. Aquellas pacientes con radioterapia pelviana exclusiva tuvieron mayor riesgo de diseminación a distancia (32\% vs $25 \%$ ). Sin embargo, la toxicidad fue mayor para las pacientes con irradiación lumboaórtica.

La EORTC (European Organization for Research and Treatment of Cancer) también desarrollo un protocolo similar para evaluar los resultados de radioterapia profiláctica a nivel lumboaórtico (30); se comparó la radioterapia pelviana exclusiva versus la radioterapia de pelvis más lumboaórtica en 441 pacientes estadio IIB y III. En este trabajo fueron excluidas las pacientes con evidencia de ganglios positivos a nivel lumboaórtico, pero el $74 \%$ eran pacientes con enfermedad avanzada en pelvis (IIB distal o IIIB). La sobrevida a 4 años no presentó diferencias estadísticamente significativas (50\% versus $53 \%$ ). Sin embargo, la recidiva sistémica fue 2,8 veces mayor en las pacientes que recibieron radioterapia pelviana exclusiva. Estos resultados se pueden explicar por el hecho de que estas pacientes tenían una enfermedad pelviana avanzada, por lo cual la ventaja de la irradiación lumboaórtica queda enmascarada por la falla de control del tumor pelviano, (recidiva local entre 49 y $51 \%$ para radioterapia pelviana y pelviana mas lumboaórtica, respectivamente). Como conclusión de estos trabajos podemos decir que la radioterapia lumboaórtica profiláctica puede cambiar el patrón de diseminación del cáncer de cuello uterino, y por lo tanto aumentar la sobrevida. Sin embargo, para aquellas pacientes con una enfermedad avanzada a nivel de la pelvis, este aumento de la sobrevida está supeditado al control del tumor primario, lo que se hace más evidente en las pacientes con etapa IIIB. Un mayor beneficio se vería en aquellas pacientes con tumores pelvianos menos avanzados como las incluidas en el protocolo RTOG 79-20 (11).

\section{CONCLUSIÓN}

La diseminación del $\mathrm{CaCu}$ es relativamente predecible y ordenada, por lo que se puede conocer con relativa precisión el riesgo de compromiso ganglionar lumboaórtico en cada etapa de la enfermedad. La irradiación lumboaórtica se ha demostrado efectiva, tanto en pacientes con una enfermedad evidente como en aquellas donde el riesgo de metástasis a este nivel es elevado. Sin embargo, como se desprende de los dos trabajos randomizados, son las pacientes con enfermedad pelviana con- trolable y con elevado riesgo de tener compromiso ganglionar lumboaórtico las que más se benefician de dicho tratamiento (etapa IA-B, IIA-B con ganglios positivos en la pelvis). En el otro extremo se encuentran dos grupos de pacientes, aquellas con ganglios positivos lumboaórticos (alto riesgo de diseminación a distancia) y aquellas con enfermedad pelviana masiva (IIIB), en donde el tratamiento de radiación lumboaórtica no reflejaría una mejoría en la sobrevida debido a una alta posibilidad de recidiva a distancia en las primeras o por una falla en el control pelviano en las segundas.

\section{BIBLIOGRAFÍA}

1. Donoso E, Cuello M, Villarroel L. Reducción de la mortalidad por cáncer de cuello uterino en Chile. Rev Chil Obstet Ginecol 2006;71:307-12

2. Pérez C. Uterine Cervix. In Pérez C, Brady L. Principles \& Practice of Radiation Oncology. $3^{\mathrm{a}}$ ed. Lippincott-Raven. Philadelphia. 1998.

3. Hughes RR, Brewington KC, Hanjani P, Photopulos $\mathrm{G}$, Dick $\mathrm{D}$, et al. Extended field irradiation for cervical cancer based on surgical staging. Gynecol Oncol 1980;9:153-61.

4. Ballon SC, Berman ML, Lagasse LA, Petrilli E, Castaldo T. Survival after extraperitoneal pelvic and paraaortic lymphadenectomy and radiation therapy in cervical carcinoma Obstet Gynecol 1981;57:90-5.

5. Piver MS, Barlow JJ, Krishnamsetty R. Five-year survival (with no evidence of disease) in patients with biopsy-confirmed aortic node metastases from cervical carcinoma. Am J Obstet Gynecol 1981:139:575-8.

6. Welander CE, Pierce VK, Nori D, Hilaris BS, Kosloff $\mathrm{C}$, et al. Pretreatment laparotomy in carcinoma of the cervix. Gynecol Oncol 1981:12:336-47.

7. Potish RA, Twiggs LB, Okagaki KA. Therapeutic implications of the natural history of advanced cervical cancer as defined by pretreatment surgical staging. Cancer 1985;56:956-60.

8. La Polla JP, Schlaerth JB, Gaddis O, Morrow C. The influence of surgical staging on the evaluation and treatment of patients with cervical carcinoma. Gynecol Oncol 1986;24:194-9.

9. Vigliotti AP, Wen BC, Hussey DH, DoornobosJ, Staples J, Jani S. Extended field irradiation for carcinoma of the uterine cervix with positive periaortic nodes. Int J Radiat Oonco Biol Phys 1992;23:501-9.

10. Buchsbaum HJ. Extrapelvic lymph node metastases in cervical carcinoma. Am J Obstet Gynecol 1979;133:814-24.

11. Grigsby PW, Haydon K, Match DG, Kim R, Eifel P. Longterm follow-up of RTOG 92-10: cervical cancer with positive para-aortic lymph nodes. Int $\mathrm{J}$ Radiat Oncol Biol Phys 2001;49:733-8.

12. Rotman M, Thomas $\mathrm{P}$, Kwan C, Clery M, Marcial V, 
Grigsby $\mathrm{P}$, et al. Prophylactic extended-field irradiation of para-aortic lymph nodes in stages IIB and bulky IB and IIA cervical carcinomas. Ten-year treatment results of RTOG 79-20. JAMA 1995;274:387-93.

13. Regules E, Borgno L, Scaniello V. Sistematización de las cadenas nodales linfáticas de la pelvis femenina. Int J Morphol 2005;23:353-62.

14. Rotman M, Aziz H, Eifel P. Irradiation of pelvic and paraortic nodes in carcinoma of the cervix. Semin Radiat Oncol 1994;4:23-9.

15. Fuller AF, Elliott N, Koskoff C, Lewia J. Lymph node metastases from carcinoma of the cervix, stages IB and IIA: implications for prognosis and treatment. Gynecol Oncol 1982;13:165-74.

16. Finan MA, De Cesare S, Fiorica JV, Chambers R, Hoffman M, Kline R, et al. Radical hysterectomy for stage IB1 vs IB2 carcinoma of the cervix: does the new staging system predict morbidity and survival? Gynecol Oncol 1996;62:139-47.

17. Inoue T, Childara T, Morita K. The prognostic significance of the size of the largest nodes in metastatic carcinoma from the uterine cervix. Gynecol Oncol 1984;19:187-93.

18. Samlal RA, Van der Velden J, Ten Kate, Schilthuis M, Hart A, lammers F. Identification of high-risk groups among node-positive patients with stage IB and IIA cervical carcinoma. Gynecol Oncol 1997;64:463-7.

19. Monagham JM, Ireland D, Mor-Yosef S, Pearson S, Lopez A, Sinha D, et al. Role of centralization of surgery in stage IB carcinoma of the cervix: a review of 498 cases. Gynecol Oncol 1990;37:206-9.

20. Creasman WT, Soper JT, Clarke-Pearson D. Radical hysterectomy as therapy for early carcinoma of the cervix. Am J Obstet Gynecol 1986;155:964-9.

21. Artman LE, Hoskins WJ, Bibro MC, Heller P, Weiser E, Barnhill D, et al. Radical hysterectomy and pelvic lymphadenectomy for stage IB carcinoma of the cervix: 21 years experience. Gynecol Oncol 1987;28:8-13.

22. Zander J, Baltzer J, Lohe KJ, Ober K, Kaufman C. Carcinoma of the cervix: an attempt to individualize treatment. Results of a 20-year cooperative study. Am J Obstet Gynecol 1981;139:752-9.

23. Timmer PR, Aalder JC, Bouma J. Radical surgery after preoperative intracavitary radiotherapy for stage IB and IIA carcinoma of the uterine cervix. Gynecol Oncol 1984;18:206-12.

24. Nelson JN Jr, Boyce J, Macaset M, Lu T, BhorquezJ Nicastri $A$, et al. Incidence, significance, and followup of para-aortic lymph node metastases in late invasive carcinoma of the cervix. Am J Obstet Gynecol 1977;128:336-40.

25. Delgado G, Chun B, Caglar H, Bepko F. Paraaortic lymphadenectomy in gynecologic malignancies confined to the pelvis. Obstet Gynecol 1977;50:418-23.
26. Piver MS. The value of pretherapy para-aortic lymphadenectomy for carcinoma of the cervix uteri. Surg Gynecol Obstet 1977;145:17-8.

27. Sudarsanam A, Charyuluk B, Belinsom J, Averette $H$, Goldberg $\mathrm{M}$, et al. Influence of exploratory celiotomy on the management of carcinoma of the cervix. A preliminary report. Cancer 1978;41:1049-53.

28. Berman ML, Keyes H, Creasman W, DiSaia P, Bundy $\mathrm{B}$, Blessing $\mathrm{J}$, et al. Survival and patterns of recurrence in cervical cancer metastatic to periaortic lymph nodes - a Gynecologic Oncology Group study. Gynecol Oncol 1984;19:8-16.

29. Rodtman M, Moon S, John M. Extended field paraaortic radiation in cervical carcinoma the case for prophylactic treatment. Int J Radiat Oncol Biol Phys 1978;4:795-9.

30. Haie C, Pejovic MH, Gerbaulet A, Horiot J, Purquier $\mathrm{H}$, Delouche J, et al. Is Prophylactic para-aortic irradiation worthwhile in the treatment of advanced cervical carcinoma? Results of a controlled clinical trial of the EORTC radiotherapy group. Radiother Oncol 1988;11:101-12.

31. Lovecchio JL, Averette HE, Donato D, Bell J. 5-year survival of patients with periaortic nodal metastases in clinical stage IB and IIA cervical carcinoma. Gynecol Oncol 1989;34:43-5.

32. Stryker JA, Mortel R. Survival following extended field irradiation in carcinoma of cervix metastatic to paraaortic lymph nodes. Gynecol Oncol 2000;79:399-405.

33. Yildilrin Y, Schiralis S, Avici ME, YImaz C, Ertopcu K, Tinar S, et al. Integrated PET/CT for the evaluation of para-aortic nodal metastasis in locally advanced cervical cancer patients with negative conventional CT findings. Gynecol Oncol 2008;108;154-9.

34. Vongtama V, Piver SM, Tsukada Y Barlow J, and Webster J: Para-aortic node irradiation in carcinomas. Cancer 1974;34:169-74

35. Weiser EB, Bundy BN, Hoskins WJ, Whittington RR, DiSaia PJ, Curry SL, et al Extraperitoneal versus transperitoneal selective paraortic lymphadenectomy in the pretreatment surgical staging of advanced cervical carcinoma - a GOG study. Gynecol Oncol 1989;33:283-9.

36. Cunningam MJ, Duntom CJ, Corn B, Numoff J, Morgan M, King S, et al. Extended-field radiation therapy in early-stage cervical carcinoma: survival and complications. Gynecol Oncol 1991;43:51-54

37. Grigsby P, Singh A, Siegel B, Siegel B, Dehdashti F, Rader J. y col. Lymph node control in cervical cancer Int J Radiat Oncol Biol Phys 2004;59:706-12

38. Grigsby P, Heydon K, Mutch D Kim R, Eifel P. Longterm follow-up of RTOG 92-10: cervical cancer with positive para-aortic lymph nodes. Int $\mathrm{J}$ Radiat Oncol Biol Phys. 2001;15;51:982-7. 